\title{
L-Carnitine supplementation reduces the general fatigue of cancer patients during chemotherapy
}

\author{
HIROKI MATSUI $^{1 *}$, TAKAHIRO EINAMA ${ }^{1-3^{*}}$, SHUNSUKE SHICHI $^{1}$, RYO KANAZAWA ${ }^{1}$, \\ KAZUAKI SHIBUYA ${ }^{1}$, TAKASHI SUZUKI ${ }^{1}$, FUMIHIKO MATSUZAWA ${ }^{1}$, TAKU HASHIMOTO ${ }^{1}$, \\ SHIGENORI HOMMA ${ }^{2}$, JUNJI YAMAMOTO ${ }^{3}$, AKINOBU TAKETOMI $^{2}$ and HIRONORI ABE ${ }^{1}$ \\ ${ }^{1}$ Department of Surgery, Hokkaido Social Work Association Obihiro Hospital, Obihiro, Hokkaido 080-0805; \\ ${ }^{2}$ Department of Gastroenterological Surgery I, Hokkaido University Graduate School of Medicine, Sapporo, \\ Hokkaido 060-8638; ${ }^{3}$ Department of Surgery, National Defense Medical College, Tokorozawa, Saitama 359-8513, Japan
}

Received September 28, 2017; Accepted November 22, 2017

DOI: $10.3892 / \mathrm{mco} .2018 .1557$

\begin{abstract}
L-Carnitine (LC) plays an important role in the metabolism of fatty acids, and LC deficiency is associated with a feeling of weakness or general fatigue. Cancer patients receiving chemotherapy often develop LC deficiency, which is considered to be a factor contributing to general fatigue. The aim of the present study was to evaluate the efficacy of LC supplementation as a treatment for general fatigue in cancer patients during chemotherapy. A total of 11 cancer patients who were suffering from general fatigue during chemotherapy in our hospital between September 2014 and December 2015 were examined ( 6 cases involved adjuvant chemotherapy and 5 cases involved chemotherapy for unresectable or recurrent disease). The patients were administered $1,500 \mathrm{mg} /$ day of levocarnitine per os, and the change in mean daily fatigue from the baseline to 8 weeks was assessed using the Brief Fatigue Inventory. The change in the plasma levels of albumin and the lymphocyte counts from the baseline to 8 weeks were also assessed. LC supplementation reduced general fatigue in all cases. Moreover, LC supplementation maintained the plasma levels of albumin and lymphocyte counts during chemotherapy, and enabled patients to continue chemotherapy sequentially without dose reduction. Therefore, LC supplementation improved general fatigue in all the examined cancer patients during chemotherapy. This treatment may make improve the tolerability of chemotherapy in cancer patients by reducing general fatigue and improving the nutritional status.
\end{abstract}

Correspondence to: Dr Takahiro Einama, Department of Gastroenterological Surgery I, Hokkaido University Graduate School of Medicine, Kita-Ku, Kita 14, Nishi 7, Sapporo, Hokkaido 060-8638, Japan

E-mail: titiuehahaue@hotmail.com

*Contributed equally

Key words: L-carnitine, general fatigue, chemotherapy

\section{Introduction}

Fatigue is a common symptom in cancer patients. The rate of metastatic cancer patients with cancer-related fatigue (CRF) is $>75 \%$, and cancer survivors report that, even if their condition improves after treatment, fatigue often persists for months or even years. Cancer patients state that fatigue is one of the most distressing symptoms associated with cancer, and the general condition during cancer treatment is difficult to manage with medications (1).

The pathophysiology of fatigue in cancer patients is multifactorial. Suggested mechanisms include an imbalance in energy metabolism due to tumor growth, infection, fever, or surgery, malnutrition caused by anorexia, nausea, or vomiting, and an increase in the abnormal production of substances that impair metabolic homeostasis, such as cytokines and proteolysis-inducing factors (2). Other researchers have suggested mechanisms that link fatigue to the pathophysiology of sleep disorders, major depression anemia, or hypoxia. However, there is no conclusive evidence to support the application of specific methods to reduce fatigue in cancer patients; thus, further investigation is required (1).

Carnitine, a micronutrient derived from an amino acid, is found in almost all cells of the body, and it plays an important role in energy metabolism (3). Long-chain fatty acids are transported by carnitine across the membranes of mitochondria in muscle cells, and $\beta$-oxidation converts fatty acids into energy in the form of adenosine triphosphate. Additionally, carnitine also transports fatty acids out of the mitochondria (3). Carnitine reabsorption is regulated through the proximal tubules of the kidney. Skeletal and cardiac muscles use fatty acids as their primary source of energy; thus, carnitine deficiency is associated with low energy levels and muscle weakness (4).

Carnitine homeostasis is regulated by oral intake, synthesis and renal reabsorption. In humans, carnitine is produced in the liver and kidneys, stored in skeletal muscle and excreted in the urine. Certain cytotoxic chemotherapies interfere with the homeostasis and reabsorption of carnitine (5-7). Nausea, vomiting and anorexia caused by cancer treatment may reduce the oral intake of food, which may lead to low plasma levels of carnitine (8). 
Recent studies demonstrated that L-carnitine (LC) supplementation in patients with cancer was effective in reducing fatigue $(4,5)$ and increasing the lean body mass and appetite (9). However, the type of patients who may benefit from LC supplementation has not been fully elucidated. The aim of the present study was to monitor fatigue in cancer patients who received chemotherapy, adjuvant chemotherapy or treatment for metastatic disease, and to evaluate changes in fatigue prior to and following LC supplementation.

\section{Patients and methods}

Patients. The present study was performed with the approval of the Internal Review Board on ethical issues of Hokkaido Social Work Association Obihiro Hospital. A total of 11 patients who underwent chemotherapy in our hospital between September 2014 and December 2015 and experienced fatigue during chemotherapy were retrospectively reviewed. The inclusion criteria were as follows: i) Pathological diagnosis of cancer; ii) the patients must have been informed of their cancer diagnosis; iii) administration of at least one cycle of a chemotherapeutic regimen; iv) Eastern Cooperative Oncology Group performance status of 0 or 1 ; v) the patients had to be able to understand and complete the questionnaires; and vi) no severe mental or cognitive disorders.

Fatigue measurements. The Brief Fatigue Inventory (BFI) was selected to assess fatigue, as it is a multidimensional, independent, commonly used scale for the clinical assessment of fatigue. The BFI consists of 9 items, using a numerical scale of $0-10$ on a single page. The global score for the BFI is calculated as the mean value of these 9 items and categorized as follows: Scores of 1-3, mild; scores of 4-6, moderate; and scores of 7-10, severe $(10,11)$.

Treatment plan with LC. Based on the current knowledge on carnitine use, its metabolism, and treatment of deficiency, a daily oral dose of $1.5 \mathrm{~g}$ LC divided into in three single doses of $0.5 \mathrm{~g}$ was administered for 8 weeks, preferably during or following meals.

Study design. The enrolled patients were evaluated for fatigue at the baseline and after 2 weeks of LC supplementation. Following baseline evaluation, the patients started treatment with $1.5 \mathrm{~g}$ of oral LC three times daily for 8 weeks. During treatment, the patients were monitored every 2 weeks with physical examination, medical history and blood chemistry tests. The patients were stratified into two categories according to chemotherapy, namely the adjuvant chemotherapy (Adj.) and unresectable or recurrence (Rec.) groups.

Statistical analysis. The patient records were entered into our database and completed with information obtained from a retrospective review of hospital and physician records. Statistical analysis was performed using the JMP 13.0.0 software package (SAS Institute, Inc., Tokyo, Japan).

\section{Results}

Patient demographics and tumor characteristics. The mean patient age was 67.5 years [standard deviation (SD) \pm 7.3 years].

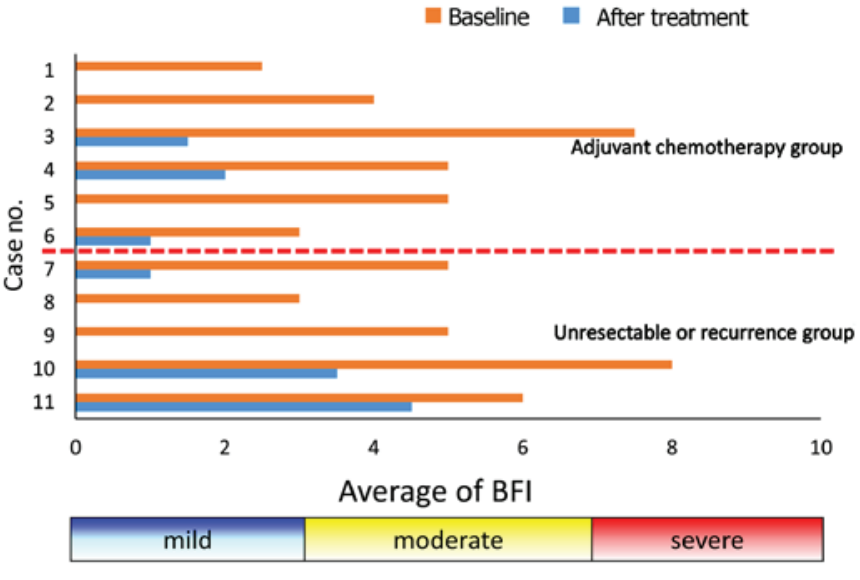

Figure 1. Changes in the BFI score during 8 weeks in 11 chemotherapy patients treated with L-carnitine. In all the patients, the pre-treatment scores were significantly higher compared with the post-treatment scores (mean pre-BFI: $4.9 \pm 1.8$; post-BFI: $1.2 \pm 1.6$; $\mathrm{P}<0.0001$ ). BFI, Brief Fatigue Inventory.

Of the 11 patients, 4 were men and 7 were women. The location of the cancer was colorectal in 8 patients, pancreatic in 2 patients, and in the biliary tract in 1 patient. A total of 6 cases received adjuvant chemotherapy and 5 cases received treatment for recurrent or metastatic cancer. The chemotherapy regimens for colorectal cancer included FOLFIRI + bevacizumab for 3 patients, FOLFOX for 2 patients, FOLFOX + bevacizumab for 1 patient, FOLFIRI + panitumumab for 1 patient and TAS-102 for 1 patient. The chemotherapy regimens used for pancreatic cancer were S-1 and gemcitabine + nab-paclitaxel. The chemotherapy administered for biliary tract cancer was gemcitabine + cisplatin (Table I).

Assessment of fatigue. The BFI scores for all patients in the present study are presented in Fig. 1. In all the patients, the pre-treatment scores were significantly higher compared with the post-treatment scores (mean pre-BFI: $4.9 \pm 1.8$ vs. post-BFI: $1.2 \pm 1.6 ; \mathrm{P}<0.0001)$. The mean $\pm \mathrm{SD}$ changes in the BFI scores were $-3.8 \pm 0.81$ and $-3.6 \pm 1.2$ in the Adj. and Rec. groups, respectively. The difference in the mean change in the scores between the groups was -0.2 (not statistically significant). The mean \pm SD BFI scores after treatment were $0.75 \pm 0.88$ in the Adj. and 1.8 2.1 in the Rec. group.

Nutritional assessment. The mean \pm SD changes in the levels of albumin were $0.017 \pm 0.14 \mathrm{mg} / \mathrm{dl}$ in the Adj. group and $-0.20 \pm 0.14 \mathrm{mg} / \mathrm{dl}$ in the Rec. group. The difference was not statistically significant in either group (Fig. 2).

The mean \pm SD lymphocyte counts in the Adj. group were 939.7 $\pm 478.5 / \mu 1$ pre-treatment and $1,085.5 \pm 478.5 / \mu 1$ post-treatment. A total of 4 patients exhibited increased lymphocyte counts after treatment. The mean \pm SD lymphocyte counts in the Rec. group were $1,291.6 \pm 637.7 / \mu \mathrm{l}$ pre-treatment and $1,169.2 \pm 554.0 / \mu 1$ post-treatment. The difference was not statistically significant in either group (Fig. 3).

Safety. According to the National Cancer Network-Common Terminology Criteria, no adverse events potentially associated with LC were reported for any patient during this study. 
Table I. Characteristics of the patients enrolled in the present study.

\begin{tabular}{rcllll}
\hline Case no. & Age, years & Sex & Primary & \multicolumn{1}{c}{ Regimen } & Adj. or Rec. \\
\hline 1 & 73 & M & Bile duct & GEM + CDDP & Adj. \\
2 & 68 & M & Rectum & FOLFOX & Adj. \\
3 & 69 & F & Pancreas & TS-1 & Adj. \\
4 & 52 & F & Colon & FOLFOX & Adj. \\
5 & 65 & F & Colon & FOLFIRI + Bmab & Adj. \\
6 & 74 & F & Rectum & FOLFIRI + Bmab & Adj. \\
7 & 68 & F & Pancreas & GEM + nab-paclitaxel & Rec. (bone) \\
8 & 74 & F & Colon & FOLFOX + Bmab & Rec. (liver) \\
9 & 64 & M & Colon & FOLFIRI + Pmab & Rec. (peritoneal) \\
10 & 77 & F & Colon & FOLFIRI + Bmab & Rec. (lung, peritoneal) \\
11 & 59 & M & Rectum & TAS-102 & Rec. (liver, peritoneal) \\
\hline
\end{tabular}

Adj., adjuvant chemotherapy; Rec., recurrent or metastatic cancer; Bmab, bevacizumab; Pmab, panitumumab; M, male; F, female; GEM, gemcitabine; CDDP, cisplatin.

Adjuvant chemotherapy group

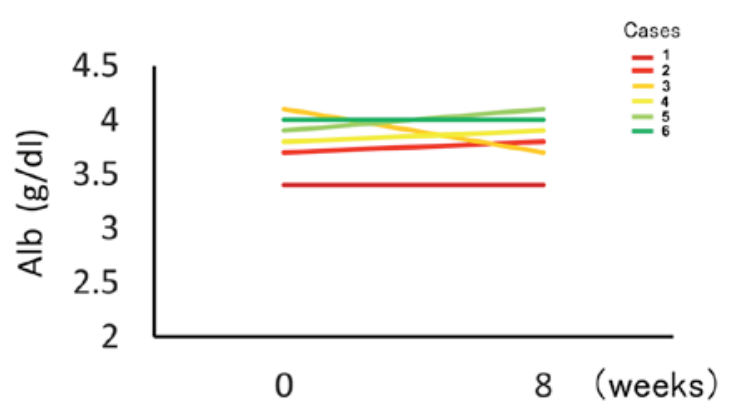

Unresectable or recurrence group

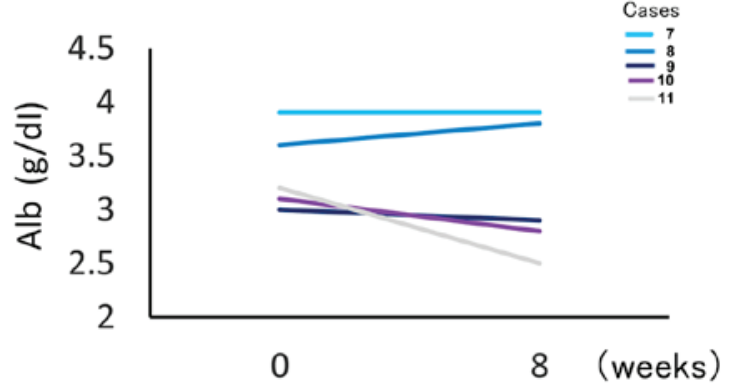

Figure 2. Effect of L-carnitine supplementation on serum albumin $>8$ weeks in chemotherapy patients. The mean \pm standard deviation changes in levels of albumin were $0.017 \pm 0.14 \mathrm{mg} / \mathrm{dl}$ in the adjuvant chemotherapy (Adj.) Group and $-0.20 \pm 0.14 \mathrm{mg} / \mathrm{dl}$ in unrecurrent (Rec.) group. The difference was not statistically significant in either group.

Adjuvant chemotherapy group

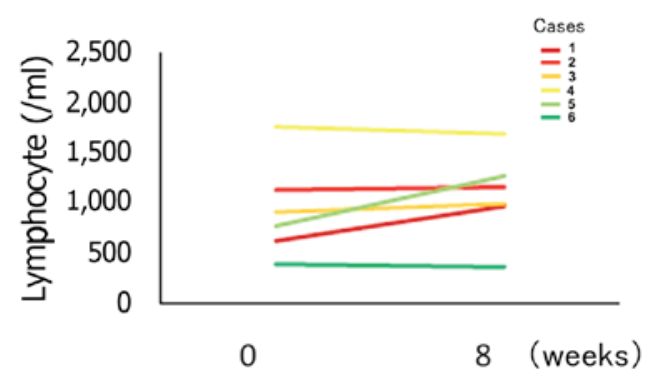

Unresectable or recurrence group

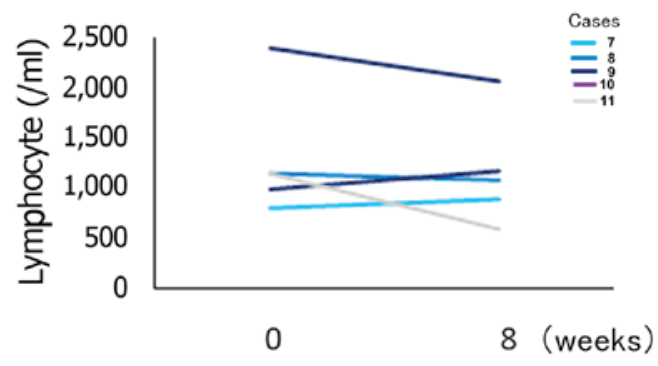

Figure 3. Effect of L-carnitine supplementation on the lymphocyte count $>8$ weeks in chemotherapy patients. The mean \pm standard deviation (SD) lymphocyte counts in the adjuvant chemotherapy (Adj.) group were 939.7 $\pm 478.5 / \mu 1$ pre-treatment and 1,085.5 $\pm 478.5 / \mu 1$ post-treatment. The mean \pm SD lymphocyte counts in the unrecurrent (Rec.) group were 1,291.6 $\pm 637.7 / \mu 1$ pre-treatment and 1,169.2 $\pm 554.0 / \mu 1$ post-treatment. The difference was not statistically significant in either group.

Chemotherapy was well-tolerated by all patients during this study.

\section{Discussion}

In the present study, hat LC supplementation reduced fatigue in all cancer patients. There was no significant difference in the mean change of BFI scores between the Adj. and Rec. groups. Based on these data, it may be suggested that chemotherapy-induced damage of the carnitine system and secondary deficiency of this molecule may cause fatigue due to impaired energy metabolism $(12,13)$. Thus, restoration of the carnitine pool may alleviate the fatigue of cancer patients (14). In the Rec. group, fatigue was still marked compared with the Adj. 
group after LC supplementation, as cytokine production from cancer cells and pro-inflammatory cytokines from cancer patients may also contribute to the fatigue (15). To the best of our knowledge, this is the first study to investigate therapeutic intervention with carnitine in the Adj. and Rec. patient groups. The results were encouraging, as LC supplementation reduced chemotherapy-induced fatigue in all patients who received this treatment.

Second, it was demonstrated that LC supplementation improved the nutritional status of the patients. In addition, all Adj. group patients continued chemotherapy sequentially, and did not require a reduction in the dose of the chemotherapy regimen. LC supplementation also leads to an improved nitrogen balance, either due to increased protein synthesis or abrogation of the inflammatory processes under pathological conditions, prevents oxidative stress, and improves mitochondrial function (16).

The beneficial effect of LC supplementation on mechanisms involved in skeletal muscle loss under pathological conditions may explain its anti-catabolic effects and/or the improvement of fatigue-related parameters following LC supplementation in patients with chronic kidney disease $(17)$, cancer $(5,9,18,19)$, and hepatitis C and hepatic encephalopathy (20).

LC supplementation improved general fatigue in all cancer patients during chemotherapy. LC may improve the ability of cancer patients to cope with chemotherapy by reducing general fatigue and improving the nutritional status. The limitations of the present study included its design (single-institution, non-randomized study), the limited number of included patients, and the fact that carnitine levels in the plasma were not measured. Despite these limitations, our findings may provide evidence to support the efficacy of LC supplementation in controlling CRF. However, further research is required to confirm our findings. A randomized, double-blind, placebo-controlled trial is currently being planned to demonstrate the efficacy of LC supplementation, based on previously published LC supplementation articles $(18,21)$.

\section{Acknowledgements}

The authors would like to thank assistant secretary Ms. Sachiko Saito for her support.

\section{Competing interests}

The authors declare that they have no competing interests.

\section{References}

1. Mock V, Atkinson A, Barsevick AM, Berger AM, Cimprich B Eisenberger MA, Hinds P, Kaldor P, Otis-Green SA and Piper BF: Cancer-related fatigue. Clinical practice guidelines in oncology. J Natl Compr Canc Netw 5: 1054-1078, 2007.

2. Glaspy J: Anemia and fatigue in cancer patients. Cancer 92 (Suppl 6): S1719-S1724, 2001.

3. Foster DW: The role of the carnitine system in human metabolism. Ann N Y Acad Sci 1033: 1-16, 2004.

4. Cruciani RA, Dvorkin E, Homel P, Culliney B, Malamud S, Shaiova L, Fleishman S, Lapin J,Klein E,Lesage P, et al: L-carnitine supplementation for the treatment of fatigue and depressed mood in cancer patients with carnitine deficiency: A preliminary analysis. Ann N Y Acad Sci 1033: 168-176, 2004.
5. Graziano F, Bisonni R, Catalano V, Silva R, Rovidati S, Mencarini E, Ferraro B, Canestrari F, Baldelli AM, De Gaetano A, et al: Potential role of levocarnitine supplementation for the treatment of chemotherapy-induced fatigue in non-anaemic cancer patients. Br J Cancer 86: 1854-1857, 2002.

6. Waldner R, Laschan C, Lohninger A, Gessner M, Tüchler H, Huemer M, Spiegel W and Karlic H: Effects of doxorubicin-containing chemotherapy and a combination with L-carnitine on oxidative metabolism in patients with non-Hodgkin lymphoma. J Cancer Res Clin Oncol 132: 121-128, 2006.

7. Bianchi G, Vitali G, Caraceni A, Ravaglia S, Capri G, Cundari S, Zanna C and Gianni L: Symptomatic and neurophysiological responses of paclitaxel- or cisplatin-induced neuropathy to oral acetyl-L-carnitine. Eur J Cancer 41: 1746-1750, 2005.

8. Hockenberry MJ, Hooke MC, Gregurich M and McCarthy K: Carnitine plasma levels and fatigue in children/adolescents receiving cisplatin, ifosfamide, or doxorubicin. J Pediatr Hematol Oncol 31: 664-669, 2009.

9. Gramignano G, Lusso MR, Madeddu C, Massa E, Serpe R. Deiana L, Lamonica G, Dessì M, Spiga C, Astara G, et al: Efficacy of 1-carnitine administration on fatigue, nutritional status, oxidative stress and related quality of life in 12 advanced cancer patients undergoing anticancer therapy. Nutrition 22: 136-145, 2006.

10. Mendoza TR, Wang XS, Cleeland CS, Morrissey M, Johnson BA, Wendt JK and Huber SL: The rapid assessment of fatigue severity in cancer patients: Use of the brief fatigue inventory. Cancer 85: 1186-1196, 1999.

11. Okuyama T, Wang XS, Akechi T, Mendoza TR, Hosaka T, Cleeland CS and Uchitomi Y: Validation study of the Japanese version of the brief fatigue inventory. J Pain Symptom Manage 25: 106-117, 2003.

12. Dodson WL, Sachan DS, Krauss S and Hanna W: Alterations of serum and urinary carnitine profiles in cancer patients: Hypothesis of possible significance. J Am Coll Nutr 8: 133-142, 1989.

13. Peluso G, Nicolai R, Reda E, Benatti P, Barbarisi A and Calvani M: Cancer and anticancer therapy-induced modifications on metabolism mediated by carnitine system. J Cell Physiol 182: 339-350, 2000.

14. Brass EP, Adler S, Sietsema KE, Hiatt WR, Orlando AM and Amato A; CHIEF Investigators: Intravenous L-carnitine increases plasma carnitine, reduces fatigue and may preserve exercise capacity in hemodialysis patients. Am J Kidney Dis 37: 1018-1028, 2001.

15. McNeil C: Cancer fatigue: One drug fails but more are in the pipeline. J Natl Cancer Inst 93: 892-893, 2001.

16. Ringseis R, Keller J and Eder K: Mechanisms underlying the anti-wasting effect of L-carnitine supplementation under pathologic conditions: Evidence from experimental and clinical studies. Eur J Nutr 52: 1421-1442, 2013.

17. Fatouros IG, Douroudos I, Panagoutsos S, Pasadakis P, Nikolaidis MG, Chatzinikolaou A, Sovatzidis A, Michailidis Y, Jamurtas AZ, Mandalidis D, et al: Effects of L-carnitine on oxidative stress responses in patients with renal disease. Med Sci Sports Exerc 42: 1809-1818, 2010.

18. Cruciani RA, Dvorkin E, Homel P, Culliney B, Malamud S, Lapin J, Portenoy RK and Esteban-Cruciani N: L-carnitine supplementation in patients with advanced cancer and carnitine deficiency: A double-blind, placebo-controlled study. J Pain Symptom Manage 37: 622-631, 2009.

19. Iwase S, Kawaguchi T, Yotsumoto D, Doi T, Miyara K, Odagiri H, Kitamura K, Ariyoshi K, Miyaji T, Ishiki H, et al: Efficacy and safety of an amino acid jelly containing coenzyme Q10 and L-carnitine in controlling fatigue in breast cancer patients receiving chemotherapy: A multi-institutional, randomized, exploratory trial (JORTC-CAM01). Support Care Cancer 24: 637-646, 2016.

20. Malaguarnera M, Vacante M, Giordano M, Pennisi G, Bella R, Rampello L, Malaguarnera M, Li Volti G and Galvano F: Oral acetyl-L-carnitine therapy reduces fatigue in overt hepatic encephalopathy: A randomized, double-blind, placebo-controlled study. Am J Clin Nutr 93: 799-808, 2011.

21. Cruciani RA, Zhang JJ, Manola J, Cella D, Ansari B and Fisch MJ: L-carnitine supplementation for the management of fatigue in patients with cancer: An eastern cooperative oncology group phase III, randomized, double-blind, placebo-controlled trial. J Clin Oncol 30: 3864-3869, 2012. 